\title{
FOCUS ON BOVINE MASTITIS: KNOWLEDGE INTO PRACTICE
}

EDITORS

Luke O'Grady and Michael Doherty

UCD Herd Health Group,

School of Agriculture, Food Science and Veterinary Medicine

University College Dublin

Mastitis continues to be a cause of significant economic loss to the dairy industry internationally. Despite significant advances in our understanding of the disease, clinical and sub-clinical mastitis remains a problem in dairy herds and prevalence rates in many countries remain similar to those published decades ago. In the complex milieu of the modern dairy farm, it is the interaction of production diseases including mastitis, their relationship with nutritional strategy, housing, environment and the fundamental influence of social and attitudinal factors that make prevention and control such a challenge. Thus, dairy cows are eight times more likely to develop mastitis if they suffered milk fever at parturition. Mastitis is the most costly disease of cattle; it impacts on animal health and welfare, on market image, on profitability and, critically, on the quality of life of dairy farmers. Mastitis is stressful for both cows and farmers. Importantly, clinical mastitis represents only the 'tip of the iceberg' and it is the significance of the sub-clinical disease that is frequently underestimated.

While there will always be more to learn about mastitis at the cellular and molecular levels, a significant body of knowledge already exists, which should allow us to optimally manage and prevent the disease. The scientific knowledge base has been rapidly increasing, but there has been inadequate emphasis on the application of existing knowledge to the development of disease management systems or on increasing the capacity for knowledge uptake. One of the challenges facing the Irish dairy industry will be the development, implementation and economic assessment of practical solutions to mastitis control. Addressing this challenge will require an integrated, partnership approach involving the farmer and the veterinarian along with Teagasc and other agricultural advisors.

The special supplement of the Irish Veterinary Journal, from which these abstracts are taken, is a compilation of peer-reviewed papers presented by leading international scientists at a two-day symposium, sponsored by Alltech, entitled 'Focus on Mastitis: Knowledge into Practice' and hosted by the Herd Health Group at University College Dublin. The supplement is available to download on www. irishveterinaryjournal.com The various topics covered include global perspectives on the dairy industry, potential applications of HACCPlike approaches to control, exploration of the contagious-environmental mastitis paradigm, therapeutic strategies and nutritional influences on the immune response. We hope that this special supplement will be regarded as an authoritative learning resource by veterinarians, farmers, advisors, researchers and undergraduate students and, given the recent launch of the Animal Health Ireland initiative by the Minister for Agriculture, Fisheries and Food, we believe that the material provides a topical, timely and authoritative review on one of the priority issues to be addressed by that important national herd health initiative. 